$\frac{46 / 8.95}{8} 95(2)$

PREPARED FOR THE U.S. DEPARTMENT OF ENERGY, UNDER CONTRACT DE-AC02-76-CHO-3073

PPPL-3124

UC-420,427

PPPL-3124

TWO-STREAM CYCLOTRON RADIATIVE INSTABILITIES DUE TO THE MARGINALLY MIRROR-TRAPPED FRACTION OF FUSION ALPHAS IN TOKAMAKS

BY

V. ARUNASALAM

JULY 1995
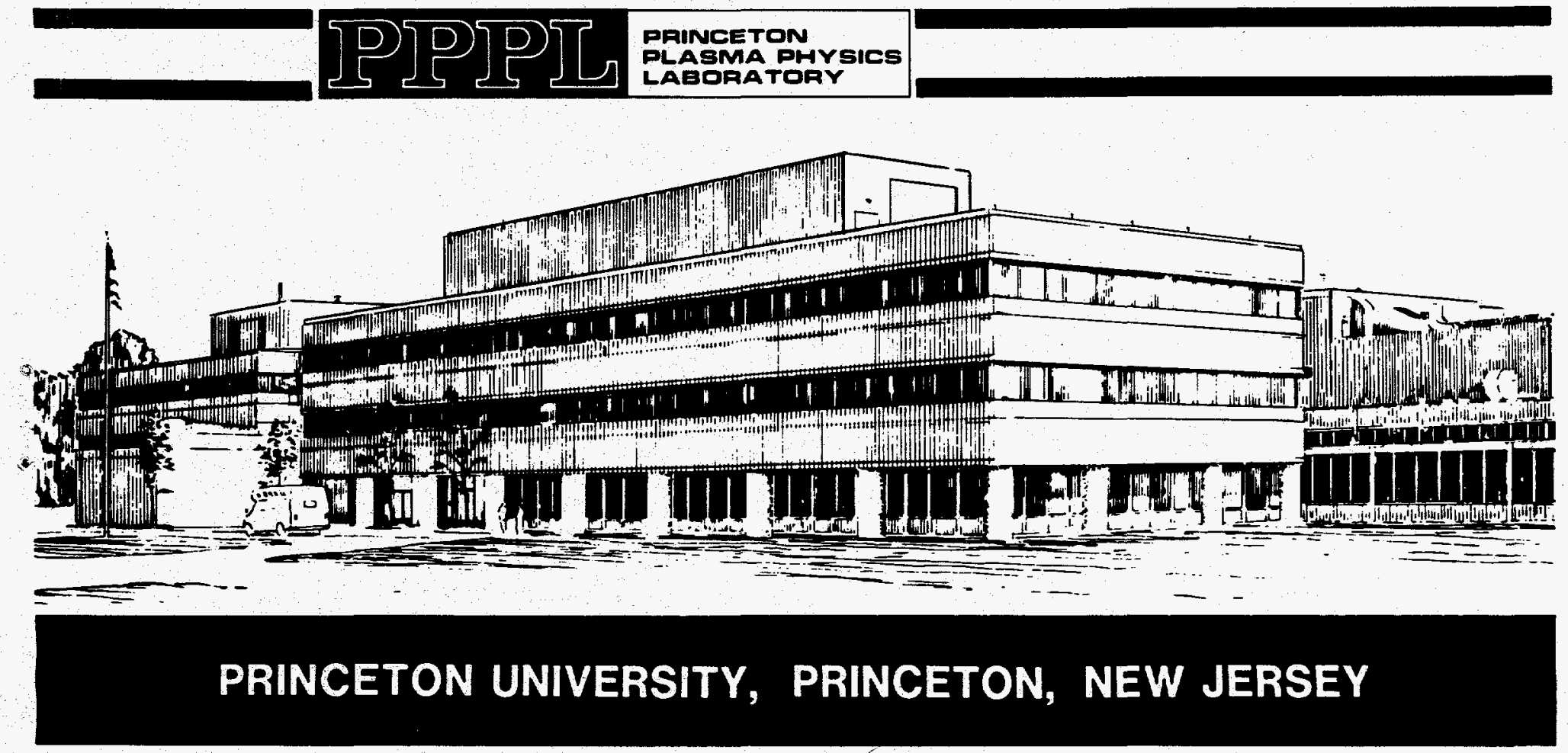


\section{NOTICE}

This report was prepared as an account of work sponsored by an agency of the United States Government. Neither the United States Government nor any agency thereof, nor any of their employees, makes any warranty, express or implied, or assumes any legal liability or responsibility for the accuracy, completeness, or usefulness of any information, apparatus, product, or process disclosed, or represents that its use would not infringe privately owned rights. Reference herein to any specific commercial produce, process, or service by trade name, trademark, manufacturer, or otherwise, does not necessarily constitute or imply its endorsement, recommendation, or favoring by the United States Government or any agency thereof. The views and opinions of authors expressed herein do not necessarily state or reflect those of the United States Government or any agency thereof.

\section{NOTICE}

This report has been reproduced from the best available copy.

Available in paper copy and microfiche.

Number of pages in this report: 19

DOE and DOE contractors can obtain copies of this report from:

Office of Scientific and Technical Information

P.O. Box 62

Oak Ridge, TN 37831 ;

(615) $576-8401$.

This report is publicly available from the:

National Technical Information Service

Department of Commerce

5285 Port Royal Road

Springfield, Virginia 22161

(703) $487-4650$ 


\section{DISCLAIMER}

Portions of this document may be illegible in electronic image products. Images are produced from the best available original document. 


\title{
TWO-STREAM CYCLOTRON RADIATIVE INSTABILITIES DUE TO THE MARGINALLY MIRROR-TRAPPED FRACTION OF FUSION ALPHAS IN TOKAMAKS
}

\author{
V. Arunasalam \\ Princeton University, Princeton Plasma Physics Laboratory \\ P.O. Box. 451, Princeton, N.J. 08543.
}

\begin{abstract}
It is shown here that the marginally mirror-trapped fraction of the newly-born fusion alpha particles in the deuterium-tritium (DT) reaction dominated tokamak plasmas can induce a two-stream cyclotron radiative instability for the fast Alfven waves propagating near the harmonics of the alpha particle cyclotron frequency $\omega^{\omega} c \alpha$. This can explain both the experimentally observed time behavior and the spatially localized origin of the fusion product ion cyclotron emission (ICE) in TFTR at frequencies $\omega \approx m \omega_{c} \alpha$.
\end{abstract}


Very recent experimental studies of ion cyclotron emission (ICE) made in the "supershot regime" [1, 2] in TFTR when high power tritium neutral heating beams were injected into a reasonably high density and high temperature background deuterium plasma yield the following observations [3]: (1) ICE at the harmonics of the cyclotron frequency of the fusion product species (i.e., alpha particles, $\omega \approx m \omega_{c} \alpha$ ) are observed immediately after the beams are turned on (i.e., at or near the beam injection time $t \approx 0$ ). (2) The time duration of these fusion product ICE peaks is $0 \leq \mathrm{t} \leq \tau_{\text {ice }} \approx 100$ to $250 \mathrm{~ms}$; while the fusion product slowing-down time from plasma center to edge [2] is $\tau_{\mathrm{S} \alpha} \approx 650$ to $130 \mathrm{~ms}$. (3) For times $t>\tau_{\text {ice }}$ these fusion product ICE subsides and are now replaced by ICE at the harmonics of the cyclotron frequency of the injected beam species [i.e., tritons, $\omega \approx m \omega_{c t}=(2 / 3) m \omega_{c \alpha}$ ] and this beam-species emission persists until the beams are turned off. (4) In all cases the observed fundamental cyclotron frequency corresponds to the value of the B field evaluated at the outer midplane plasma edge on the low field side of the torus. The dominant fusion reaction for these TFTR conditions is the DT reaction, which may be written $D+T={ }^{4} \mathrm{He}(3.6 \mathrm{MeV})+n(14.7 \mathrm{MeV})$.

It is our aim in this letter to show that during the early phase $(0 \leq t<\tau, \alpha)$ of beam injection, one can have two-stream cyclotron harmonic fast Alfven wave radiative instabilities for frequencies $\omega \approx m \omega_{c \alpha}$ induced by the small fraction of the newly born fusion alphas that are marginally mirror-trapped so that their velocities lie in the very near neighborhood of the trapped-passing boundary in the $\left(v_{\perp}, v_{\|}\right)$-plane (i.e., their velocities maintain the relation $v_{\perp} \approx v_{\|} / \sqrt{\varepsilon}$, where the mirror ratio $\varepsilon \approx \Delta B / B \approx r / R$ for tokamaks $\rangle$. These trapped-passing boundary particles are the ones with the fattest banana orbits, and they make large radial excursions sufficient to reach the outer midplane plasma edge where the experimentally observed ICE seems to originate. It should be noted that these particles make banana drift excursions only to the low field side edge near the torus midplane. As we will soon see, this two-stream cyclotron harmonic radiative instability in the region near the outer midplane 
plasma edge on the low field side of the torus is a consequence of the fact that the growth rate of these fast Alfven waves of $\omega \approx m \omega_{c \alpha}$ due to these trapped-passing boundary newly born fusion alphas (via their inverse cyclotron damping) exceeds the damping rate of these waves by the background deuterium plasma ions [4] at $\omega \approx m \omega_{c d}=m \omega_{c \alpha}$ (via the conventional cyclotron damping), and there is no contribution to emission and absorption in this low field side plasma edge region from the rest of the newly born well-confined fusion alphas that are not near this trapped-passing boundary since they do not make any banana drift excursions that are sufficiently large to reach this region. Further, this is made possible only because the cyclotron frequencies of alpha particles and deuterium ions are degenerate [4] (i.e., $\omega_{c d}=$ $\left.\omega_{c \alpha}\right)$. There are indeed two sources of free energy that drive this radiative instability or the cyclotron harmonic overstability: one coming from the ( $\perp, \mid 1)$-temperature anisotropy and the other coming from the fusion alphas directed birth velocity along $B$ (i.e., $V_{\| \alpha}$ corresponding to the alpha particles birth energy $E_{\alpha}=3.6 \mathrm{MeV}$ ). For these early times these trapped-passing "boundary alphas" velocity space distribution function $f_{b \alpha}\left(v_{\perp}, v_{\|}\right)$remains narrow enough [4] to drive the system unstable. But as time $t$ progresses $f_{b \alpha}$ broadens, first due to thermalization for $0 \leq t \leq \tau_{\alpha}$, and then due to "slowing down" for $\tau_{\alpha} \leq t<\tau_{S \alpha}$, and eventually for $t>\tau_{s \alpha}$ this distribution $f_{b \alpha}$ will totally relax towards the appropriate slowing-down distribution [5]. Here the thermalization time $\tau_{\alpha} \approx \tau_{s \alpha} / 2$ is also the heat or the energy exchange time. When $f_{b \alpha}$ broadens to sufficiently large value, one finds that this radiative instability is turned off, thus quenching the fusion product ICE in agreement with the experimental observation, and indeed we will show that the slowing-down distribution is totally stable for any emission or absorption of the cyclotron harmonic fast Alfven waves, i.e., their net absorption does exceed their net induced emission.

The method of analysis used here is the well-established standard technique of the "master 
equation approach" of non-equilibrium quantum statistical mechanics and is based on the Einstein A and B coefficients and the principle of detailed balance. In the literature other authors [6 - 8] have analysed this same problem using the standard techniques of classical plasma kinetic theory and/or plasma dispersion theory. Coppi et.al. [6], Gorelenkov and Cheng [7] have examined the fast Alfven cyclotron instability $(\mathrm{ACI})$ resulting from the resonant cyclotron interaction of the marginally trapped fusion alphas with the collective eigenmodes of the background deuterium tokamak plasma in the large aspect ratio limit. Dendy et.al. [8] have studied the magnetoacoustic cyclotron instability (MCl) resulting from this resonant cyclotron interaction of the marginally trapped fusion alphas with the modes given by the local dispersion relation for a uniform plasma with conditions comparable to those in the plasma edge. All these [6 - 8] analysis are based on kinetic and/or dispersion theory while our analysis here is based on particle-orbit theory. In general, the particle-orbit theory is much more of a physical approach and provides a better insight into the fundamental physical processes involved in a particular problem.

The velocity distribution function at the time of birth $(t=0)$ of the fusion alphas can be well approximated by a monoenergetic ring distribution $[8,4]$ with a finite $v_{\|}$such that $v_{\perp} \approx$ $v_{\|} \approx V_{\alpha}$ where $M_{\alpha} V_{\alpha} 2 / 2=E_{\alpha}$. For the "trapped-passing boundary alphas" which make banana drift excursions sufficient to reach the outer midplane plasma edge, we will take $\sqrt{ } \varepsilon V_{\perp} b \alpha=$ $V_{\| b \alpha}$, where $\varepsilon=a_{p} / R_{p}$, where $a_{p}$ and $R_{p}$ are the plasma minor and major radius, respectively. Since $V_{\alpha}{ }^{2}=V_{\perp b \alpha}{ }^{2}+V_{\| b \alpha}{ }^{2}$, we get $V_{\perp b \alpha} \approx V_{\alpha} / \sqrt{ }(1+\varepsilon)$ and $V_{\| b \alpha}=\sqrt{ } \varepsilon V_{\perp b \alpha}=V_{\alpha}[\sqrt{ } \varepsilon / \sqrt{ }(1$ $+\varepsilon)]=V_{\alpha} / \sqrt{ }\left(1+\varepsilon^{-1}\right)$. Further, since $V_{\varepsilon} v_{\perp} \approx v_{\|}$, it seems reasonable to take $\varepsilon T_{\perp} b \alpha(t) \approx$ $T_{\| b \alpha}(t)$ for these "boundary alphas". Hence for $0 \leq t<\tau_{S \alpha}$ we can approximate these boundary alphas parallel velocity space distribution function by a drifting Maxwellian of the form

$$
f_{\| b \alpha}\left(v_{\|}, t\right) \approx\left[M_{\alpha} / 2 \pi \kappa T_{\| b \alpha}(t)\right]^{1 / 2} \exp \left\{-\left[M_{\alpha} / 2 \kappa T_{\| b \alpha}(t)\right]\left[v_{\|}-v_{\| b \alpha}\right]^{2}\right\}
$$


It may be noted that when $T_{\| b \alpha}(t) \rightarrow 0, f_{\| b \alpha}\left(v_{\|}, t\right) \rightarrow \delta\left(v_{\|}-v_{\| b \alpha}\right)=f_{\| \alpha}(t=0)$ as it should. Thus the drifting Maxwell-Boltzmann distribution is a very natural evolution of an initially monoenergetic (or equivalently, an initially monovelocity) distribution during the period of thermalization with the background deuterium plasma, i.e., for times $0 \leq t \leq \tau_{\alpha}$. It may be pointed out that because of the relation $v_{\varepsilon} v_{\perp}=v_{\|}$, this parallel velocity distribution of Eq. (1) suffices to generate the perpendicular velocity distribution, and consequently, the entire distribution $f_{b \alpha}\left(v_{\perp}, v_{\|}\right) \propto f_{\| b \alpha}\left(v_{\|}\right)$. This is true only for these trapped-passing boundary particles whose $v_{\perp} \approx v_{\|} / v_{\varepsilon}$. The velocity distribution function of the background deuterium plasma ions may be written as

$$
\begin{array}{r}
f_{d}\left(v_{\perp}, v_{\|}\right)=f_{\perp d}\left(v_{\perp}\right) f_{\| d}\left(v_{\|}\right)=\left[\left(M_{d} / 2 \pi \kappa T_{\perp d}\right) \exp \left(-M_{d} v_{\perp} 2 / 2 \kappa T_{\perp d}\right)\right] \\
{\left[( M _ { d } / 2 \pi \kappa T _ { \| d } ) ^ { 1 / 2 } \operatorname { e x p } \left(-M_{d} v_{\|}{ }^{\left.\left.2 / 2 \kappa T_{\| d}\right)\right],}\right.\right.}
\end{array}
$$

Let $\eta_{\alpha}=n_{\alpha} / n_{i} \approx n_{\alpha} /\left(n_{d}+n_{\alpha}\right)$ be the total fractional density of the newiy born fusion alpha particles near the plasma center, and let $b$ be the fraction of $\eta_{\alpha}$ that forms the "trapped-passing boundary particles" for which $v_{\varepsilon} v_{\perp} \approx v_{\|}$, i.e., the trapped-passing boundary particle's fractional density is $b \eta_{\alpha}$. The fractional density of the rest (i.e., excluding these boundary alphas) of the fusion alphas is $(1-b) \eta_{\alpha}$ and these fusion alphas (which are not near the trapped-passing boundary) also have a drifting Maxwell-Boltzmann parallel velocity distribution function $f_{\| \alpha}$ of the form given by Eq. (1) with a drift velocity $V_{\| \alpha} \approx V_{\alpha}$ and a parallel temperature $T_{\| \alpha}$ and they have a regular Maxwellian perpendicular velocity distribution function with a temperature $T_{\perp} \alpha$. The fractional density of the background plasma 
deuterium ions is then $\left(1-\eta_{\alpha}\right)$. According to Rome, et.al. $[5,4]$, for times $t>\tau_{s \alpha}$, the velocity space distribution function of all these newly born fusion alphas will totally relax to the slowing-down distribution function of the form

$$
f_{\alpha}\left(v, t>\tau_{s \alpha}\right)= \begin{cases}\left(A_{o \alpha} / v_{c \alpha}\right)^{3}\left[1+\left(v / v_{c \alpha}\right)^{3}\right]^{-1} & \text { for } v \leq v_{\alpha}=\left(2 E_{\alpha} / M_{\alpha}\right)^{1 / 2} \\ 0 & \text { for } v>v_{\alpha},\end{cases}
$$

where $\left.v_{c \alpha}=(3 \sqrt{ } \pi / 4)^{1 / 3}\left(Z_{e f f} M_{e} / M_{\alpha}\right)^{1 / 3} v_{e}, A_{o \alpha}=3 /\left\{4 \pi \ln \left[1+v_{\alpha} / v_{c \alpha}\right)^{3}\right]\right\}, v_{e}=\left(2 \kappa T_{e}\right.$ $\left(M_{e}\right)^{1 / 2}$, and $Z_{e f f}=\left(\Sigma_{i} n_{i} Z_{i}^{2} / \Sigma_{i} n_{i} Z_{i}\right)$. The slowing-down time $\tau_{s \alpha}(v) \approx(3 \sqrt{ } \pi / 2)\left(M_{e} v_{e}^{3}\right.$ $\left./ M_{\alpha} A_{D}\right) \approx\left(M_{\alpha} / M_{e}\right) \tau_{e}$, where $\tau_{e}$ is the usual Spitzer electron collision time [9], and the dynamical friction parameter $A_{D} \approx 8 \pi n_{e} e^{4} Z_{\alpha}{ }^{2} \ln \Lambda / M_{\alpha}{ }^{2}$. For the TFTR plasma conditions $\ln \Lambda \approx 17$

We now consider the cyclotron emission from a dressed test charged particle (of charge $q$ and mass $M$ ) in a static confining magnetic field $B=B_{z}=B i \|$. The field particles that are responsible for "dressing" the bare test particle are the ions and electrons of the background deuterium plasma. Since we are interested in the emission and absorption of transverse electromagnetic waves at frequencies $\omega \approx m \omega_{c \alpha}=m \omega_{c d}<$ the lower hybrid frequency $\omega \mathrm{LH}$, the background index of refraction $\mu\left(=K^{1 / 2}\right.$, where $K$ is the corresponding dielectric coefficient) appropriate for dressing the bare particle emission and absorption is that corresponding to the fast Alfven waves [10], and is given by $\mu=k^{1 / 2}=c k / \omega=c / V_{A}=\left[1+\left(4 \pi n_{d} M_{d} c^{2}\right.\right.$, $\left.\left.B^{2}\right)\right]^{1 / 2}$, where $V_{A}$ is the Alfven wave phase velocity and $M_{d}$ is the mass of a deuteron. It is shown elsewhere [10] that the Einstein (quantum mechanical) spontaneous emission probability coefficient $A(m)$ for such Trubnikov extraordinary $(X)$ mode cyclotron emission is 
$A(m)=\left(4 \pi^{2} q^{2} / L^{3} K \hbar \omega\right)\left[v_{\perp} J_{m^{\prime}}(\lambda)\right]^{2} \delta\left(\omega-m \omega_{c}-k_{\|} \|_{\|}\right)$

where $\lambda=k_{\perp} \rho=k_{\perp} v_{\perp} / \omega_{c}=m k_{\perp} v_{\perp} / m \omega_{c}=m k_{\perp} v_{\perp} / k v_{A}, J_{m}{ }^{\prime}(\lambda)=\left[J_{m-1}(\lambda)-\right.$ $\left.J_{m+1}(\lambda)\right] / 2, \theta$ is the angle between $k$ and $B$, and $L^{3}$ is the plasma volume. Here we have neglected the effects of the magnetic curvature drifts. Since the $\delta$ function of Eq. (4) is a consequence of total energy and parallel momentum conservations, it is relatively easy to show $[6,10]$ that taking account of the magnetic curvature drift in this equation will result in the replacement of $\delta\left(\omega-m \omega_{c}-k_{\|} v_{\|}\right)$by $\delta\left(\omega-m \omega_{c}-k_{\|} v_{\|}-\omega_{m c d}\right)$, where $\omega_{m c d}=k \cdot v_{m c d}=$ $k_{\perp} v_{m c d}$ is the magnetic curvature drift frequency corresponding to the magnetic curvature drift velocity $v_{\text {mcd }}$. That is, all the cyclotron harmonic emission lines are upshifted by the constant amount equal to $\omega \mathrm{mcd}$. Since $\omega \mathrm{mcd}=k_{\perp}(2 \kappa T / M)(q B / M c)^{-1}(1 / 2 R)=\left(k_{\perp} \rho\right)$ $(\rho / 2 R) \omega_{C} \ll \omega_{C}$, this constant frequency-upshift due to the magnetic curvature drift effect is negligibly small. By the principle of detailed balance the rate of increase of photons in the box of volume $L^{3}$ is

$d N(\omega, k) / d t=[d N(\omega, k) / d t]_{\text {sem }}-\left\{[d N(\omega, k) / d t]_{a b}-[d N(\omega, k) / d t]_{i e m}\right\}$

where the suffixes sem, ab, and iem stand for spontaneous emission, absorption, and induced emission, respectively, and $N(\omega, k)$ is the number of photons of frequency $\omega$ and wave vector $k$ in the box of volume $L^{3}$ under study. One can show $[10]$ that $\left[d N\left(\omega \approx m \omega_{c}, k\right) / d t\right]_{s e m}=$ $<\left[L^{3} n A(m)\right]>$, and $\left\{\left[d N\left(\omega \approx m \omega_{c}, k\right) / d t\right]_{a b}-\left[d N\left(\omega \approx m \omega_{c}, k\right) / d t\right]_{i e m}\right\}=<\left[L^{3} n A(m)\right]$ $\left[\left(m \hbar \omega_{c} / M v_{\perp}\right)\left(\partial / \partial v_{\perp}\right)+\left(\hbar k_{\mid} / M\right)\left(\partial / \partial v_{\|}\right)\right]>N\left(\omega \approx m \omega_{c}, k\right)=-2 \gamma\left(\omega \approx m \omega_{c}, k\right) N(\omega \approx$ $\left.m \omega_{c}, k\right)$, where $n$ is the number density of the emitters, the angular brackets $<\cdots>$ refer to the 
statistical average over $f\left(v_{\perp}, v_{\|}\right)$, and $\gamma$ is the damping rate. Assuming that $N(\omega, k)$ is sufficiently large so that one can neglect the spontaneous emission, we find from Eq. (5) that the condition for linear radiative instability or overstability is that the induced emission exceeds the absorption or equivalently, in the language of classical plasma kinetic theory we require the net damping rate $\gamma=\gamma_{d}+\gamma_{\alpha} \leq 0$. That is,

$-2 \gamma=\Sigma_{j=d, \alpha}\left\{\left\langle\left[L^{3} n A(m)\right] \quad\left[\left(m \hbar \omega_{c} / M v_{\perp}\right)\left(\partial / \partial v_{\perp}\right)+\left(\hbar \hbar k_{\|} / M\right)\left(\partial / \partial v_{\|}\right)\right]>\right\}_{j} \quad \geq 0\right.$

Here again the angular bracket $\langle\cdots>$ of Eq. (6) refer to a statistical average over the species j charged particle velocity distribution function, and the sum is over the background deuterium plasma ions and the fusion alphas present in the region of interest. Qur immediate interest now is to examine the possible radiative instabilities in the region near the outer midplane plasma edge on the low field side of the torus. In this region near the plasma edge, the plasma ion density $n_{i}\left(r \approx a_{p}\right)=a n_{j}(r \approx 0)$, and the only fusion alphas that are present in this outer plasma edge region are these "trapped-passing boundary alphas" and their fractional density in this edge region is $\eta_{\alpha e}=n_{i} b \eta_{\alpha} / a n_{i}=\left(b \eta_{\alpha} / a\right)$. Then in this edge region the fractional deuterium plasma ion density is $\left[1-\left(b \eta_{\alpha} / a\right)\right]$. Thus, remembering that $n \propto\left[1-\left(b \eta_{\alpha} / a\right)\right]$ for the emission and absorption by the background deuterium egde plasma ions, $n \propto\left(b \eta_{\alpha} / a\right)$ for those by the "trapped-passing boundary fusion alphas," and $n \approx 0$ for the rest of the well-confined fusion alphas that are not near this trapped-passing boundary; and $v_{\perp}{ }^{-1}\left(\partial / \partial v_{\perp}\right)$ $\approx \varepsilon v_{\|}{ }^{-1}\left(\partial / \partial v_{\|}\right)$for the boundary alphas, and then making use of Eqs. (1) and (2) in Eq. (6), we obtain the necessary and sufficient condition for the two-stream cyclotron harmonic radiative instability in this edge region as 


$$
\begin{aligned}
& \left\{[ 1 - ( b \eta _ { \alpha } / a ) ] [ v _ { \perp d e } J _ { m } ( \lambda _ { d e } ) ] ^ { 2 } \left[\left(m \omega_{c \alpha} / T_{\perp d e}\right)+\left(\omega-m \omega_{c \alpha}\right) / T_{\| d e} \|\left\{\left\{k _ { \| } | ^ { - 1 } f _ { \| d } \left(v_{\|}=\right.\right.\right.\right.\right. \\
& \left.\left.v_{\alpha c p h}\right)\right\}+\left\{\left(b \eta_{\alpha} / a\right)\left[v_{\perp b \alpha} J_{m} m^{\prime}\left(\lambda_{b \alpha}\right)\right]^{2}\left[\left(\varepsilon m \omega_{c \alpha}+\omega-m \omega_{c \alpha}\right)\left\langle 1-v_{\| b \alpha^{\prime}} / v_{\alpha c p h}\right) / T_{\| b \alpha}\right]\right\} \\
& \left\{\left|k_{\|}\right|^{-1} f_{\| b \alpha}\left(v_{\|}=v_{\alpha c p h}\right)\right\} \leq 0,
\end{aligned}
$$

where the alpha particle and/or deuterium ion cyciotron phase velocity $V_{\alpha c p h}=V_{d c p h}=(\omega-$ $\left.m \omega_{c \alpha}\right) / k_{\|} ; v_{\perp d e}, \lambda_{d e}$ refer to their edge values, and we have used the fact that $\omega_{c d}=\omega_{c \alpha}$. In Eq. (7), the first term in the first curly brackets product $\{\cdots\}\{\cdots\}$ comes from the background deuterium plasma ions and this term is generally a damping term since usually $T_{\perp d}=T_{\| d}$; and the second term in the second curly brackets product $\{\cdots\}\{\cdots\}$ comes from the trapped-passing boundary alphas with $v_{\varepsilon} v_{\perp} \approx\left(a_{p} / R_{p}\right)^{1 / 2} v_{\perp} \approx v_{\|}$, and this term can lead to a growth when $v_{\alpha} \geq V_{\alpha c p h}\left[\sqrt{ }\left(1+\varepsilon^{-1}\right)\right]$,

since $V_{|| b \alpha} \approx V_{\alpha^{\prime}} / \sqrt{ }\left(1+\varepsilon^{-1}\right)$. Equation (8) only gives the necessary condition for this edge radiative instability under study, and the additional condition required to yield the necessary and sufficient condition for this radiative instability can be obtained from Eq. (7) and for $T_{\perp d e}$ $=T_{\| d e}=T_{d e}$ this sufficiency condition may be written

$\left\{\eta_{\alpha} /\left[(a / b)-\eta_{\alpha}\right]\right\} \geq\left[v_{\perp d e} J_{m}{ }^{\prime}\left(\lambda_{d e}\right) / V_{\perp b \alpha} J_{m}{ }^{\prime}\left(\lambda_{b \alpha}\right)\right]^{2}\left[T_{\| b \alpha}(t) / T_{d e}\right]\left[\left\{\left|k_{\|}\right|^{-1} f_{\| d}\left(v_{\|}=\right.\right.\right.$ $\left.\left.\left.V_{\alpha c p h}\right)\right\} /\left\{\left|k_{\|}\right|^{-1} f_{\| b \alpha}\left(v_{\|}=V_{\alpha c p h}\right)\right\}\right] / \varepsilon\left\{\left[V_{\alpha} / V_{\alpha c p h} V\left(1+\varepsilon^{-1}\right)\right]-1\right\} \geq 0$

for $\omega \approx m \omega c \alpha$, where $v_{\perp b \alpha} \approx V_{\alpha} / \sqrt{ }(1+\varepsilon)$ and $\lambda_{b \alpha}=\lambda_{\alpha} / \sqrt{ }(1+\varepsilon)$. Note, that for marginal stability, one must take the equality sign of the first inequality of Eq. (9) and, of course, this equation must yield a corresponding positive definite marginal-stability value for $\eta_{\alpha}$. If the 
right hand side of the first inequality comes out to be negative, it simply means that one cannot have any radiative instability since the fast Alfven waves under study are damped by both the newly born fusion alphas and the background deuterium plasma ions. Thus the last inequality of Eq. (9) ensures that the requirements of this equation is a necessary and sufficient condition for the radiative instability. Is is seen from Eqs. (8) and (9) that these newly born fusion alphas not only must have a sufficiently large birth velocity $V_{\alpha}$ or energy $E_{\alpha}$ so as to satisfy Eq. (8) but also must have a sufficient fractional density $\eta_{\alpha}$ so as to satisfy Eq. (9) in order to excite this radiative instability in this edge region of the plasma where the experimentally observed unstable ICE seems to originate. It should be noted that when $k_{\|} \rightarrow 0,\left\{\left|k_{\|}\right|^{-1} f_{\| d}\left(v_{\|} \mid=\right.\right.$ $\left.\left.v_{\alpha c p h}\right)\right\} \approx\left\{\left|k_{\|}\right|^{-1} f_{\| b \alpha}\left(v_{\|}=v_{\alpha c p h}\right)\right\} \rightarrow \delta(\omega-m \omega c \alpha)$. In order to account for the experimentally observed almost equal amplitude higher harmonic emission we must have $\left(k_{\perp} \rho_{\alpha}\right)>1$. For the TFTR conditions, this implies that $k \approx k_{\perp}$. Hence, for our estimation purposes we can assume that $k_{\|}$is sufficiently small so that $\left\{\left|k_{\|}\right|^{-1} f_{\| d}\left(v_{\|}=v_{\alpha c p h}\right)\right\} \approx$ $\left\{\left|k_{\|}\right|^{-1} f_{\| b \alpha}\left(v_{\|}=v_{\alpha} c p h\right)\right\} \approx \delta\left(\omega-m \omega_{c \alpha}\right)$.

In a similar way for times $t>\tau_{s \alpha}$ by making use of Eqs. (2) and (3) in Eq. (6), one can show that the necessary and sufficient condition for this radiative instability (induced in this slowed-down case by all the alphas) is

$$
\begin{aligned}
& {\left[\eta_{\alpha} /\left(1-\eta_{\alpha}\right)\right] \geq-\left[v_{\perp d} J_{m}{ }^{\prime}\left(\lambda_{d}\right) / g V_{\alpha} J_{m}\left(g \lambda_{\alpha}\right)\right]^{2}\left[f_{\| d}\left(v_{\|}=v_{\alpha c p h}\right)\right]\left(M_{\alpha} / 3 \pi A_{o \alpha} M_{d}\right)\left[v_{c \alpha}{ }^{3}\right.} \\
& \left.+\left(g^{2} v_{\alpha^{2}}+v_{\alpha c p h}\right)^{3 / 2}\right]^{2} /\left[v_{d}{ }^{2} g^{2} v_{\alpha}{ }^{2}\left(g^{2} v_{\alpha}{ }^{2}+v_{\alpha c p h}\right)^{1 / 2}\right] \geq 0
\end{aligned}
$$

where $v_{d}{ }^{2}=\left(2 \kappa T_{d} / M_{d}\right)$ and the numerical factor $g$ is somewhat less than unity. The integral over the perpendicular velocity in evaluating the angular bracket $<\cdots>$ of Eq. (6) for the slowing-down distribution function of Eq. (3) has to be done numerically. Physically, such a 
numerical integration will yield $\left\langle v_{\perp}>\approx g v_{\alpha}\right.$ with $g<1$. It is easily seen that the right hand side of the first inequality of Eq. (10) is always a negative quantity thus contradicting the second inequality of this equation. This implies that the absorption always exceeds the induced emission, and consequently, the cyclotron harmonic fast Alfven waves can never become unstable for the slowing-down distributions.

In all the Eqs. (7) - (10), we encounter the alpha particle and/or deuterium ion cyclotron harmonic phase velocity $V_{\alpha c p h}=V_{d c p h}=\left(\omega-m \omega_{c \alpha}\right) / k_{\|}=\Delta \omega / k_{\|}$. It is therefore physically instructive to understand the behavior of $v_{\alpha c p h}$. For $k_{\|}>0, v_{\alpha c p h}<0$ for $\omega<m \omega_{c \alpha}$, i.e., $\Delta \omega$ $<0$, and $V_{\alpha c p h}>0$ for $\omega>m \omega_{c \alpha}$, i.e., $\Delta \omega>0$. For $k_{\|}<0, V_{\alpha c p h}>0$ for $\omega<m \omega_{c \alpha}$, i.e., $\Delta \omega<$ 0 , and $V_{\alpha c p h}<0$ for $\omega>m \omega_{c \alpha}$, i.e., $\Delta \omega>0$. Further, $\left|V_{\alpha c p h}\right| \rightarrow 0$ for $k_{\|} \neq 0$ and $|\Delta \omega| \rightarrow 0$, and $\left|v_{\alpha c p h}\right| \rightarrow \infty$ for $|\Delta \omega| \neq 0$, and $k_{\|} \rightarrow 0$. However, for $k_{\|}=0$ and $\Delta \omega=0$ or when $k_{\|} \rightarrow 0$ and $\Delta \omega \rightarrow 0$ simultaneously, then $V_{\alpha c p h}$ is indeterminate within the framework of any conventional nonrelativistic theories [11]. The most important point to note here for our purposes is that $V_{\alpha c p h}$ can take any value, positive or negative, arbitrarily small or large, anywhere in the range $-\infty \leq V_{\alpha c p h} \leq \infty$. However, one can show that the dominant wave-particle interaction occurs for this edge radiative instability under study when $V_{\alpha c p h} \approx V_{d c p h}$ $\left(2 \kappa T_{d e} / M_{d}\right)^{1 / 2}$, since the ratio $\left[V_{d c p h} /\left(2 \kappa T_{d e} / M_{d}\right)^{1 / 2}\right]$ is the usual argument of the familiar dispersion function for the background deuterium edge plasma $[11,10]$.

According to Glasstone and Lovberg [12] the rate of production of fusion alphas may be approximated as $n_{\alpha}(r) \approx 3.7 \times 10^{-12}\left(n_{d} n_{t} / T^{2 / 3}\right) \exp \left(-20 / T^{1 / 3}\right) \mathrm{cm}^{-3} \mathrm{sec}^{-1}$, where $T$ is the ion temperature in $\mathrm{keV}$. It is reasonable to assume that $n_{d}, n_{t}$, and $T$ all vary as [1 $\left.-\left(r / a_{p}\right)^{2}\right]$ in any tokamak. Thus we see that the fusion alphas' birth profiles (whose radial variation is dominated by the exponential factor) is centrally peaked on the axis of the torus 
[2]. According to Stringer [13] and the very recent numerical calculations for JET using Stringer's theory by Cottrell,et.al. [14], one finds that approximately $10 \%$ (i.e., $b \approx 0.1$ ) of the centrally newly born alphas have a narrow range of pitch angles just near the trapped-passing boundary, and these "boundary alphas" make large radial excursions sufficient to reach the outer midplane edge where the experimentally observed ICE seems to originate. For TFTR, the value of $a=n_{i}\left(r=a_{p}\right) / n_{i}(r=0) \approx 1 \times 10^{12} \mathrm{~cm}^{-3} / 5 \times 10^{13} \mathrm{~cm}^{-3}=1 / 50=$ 0.02 , and thus in Eqs. (7) and $(9),(a / b)=0.02 / 0.1=0.2 ; T_{d}(r=0) /\left[T_{d}\left(r=a_{p}\right)=T_{d e}\right] \approx$ $25 \mathrm{keV} / 1 \mathrm{keV}=25$. Also, for TFTR, $\varepsilon \approx a_{p} / R_{p} \approx 80 \mathrm{~cm} / 245 \mathrm{~cm} \approx 0.327$; the most recent peak value [1] of $\eta_{\alpha} \approx 3 \times 10^{-3}$; by using $T_{e} \approx 9 \mathrm{keV}, T_{i} \approx 25 \mathrm{keV}, \mathrm{n}_{e} \approx n_{i} \approx 5 \times 10^{13} \mathrm{~cm}^{-3}$, $B\left(R_{p}\right) \approx 4.45 \mathrm{~T}=4.45 \times 10^{4} \mathrm{G}$, we get the alfven wave index of refraction $[10] \mu=K^{1 / 2} \approx$ 28.6, $V_{A} \approx 1.05 \times 10^{9} \mathrm{~cm} / \mathrm{s}, V_{\alpha} / V_{A}=1.25, \lambda_{\alpha} \approx 1.25 \mathrm{~m}$ for $\mathrm{k} \approx \mathrm{k}_{\perp}, v_{\alpha} \approx 1.54 \times 10^{8} \mathrm{~cm} / \mathrm{s}$, $\lambda_{d} \approx 0.15 \mathrm{~m}$ for $k \approx k_{\perp}$, the edge $\lambda_{d e} \approx 0.15 \mathrm{~m} / 5 \approx 0.03 \mathrm{~m}, \lambda_{b \alpha}=\lambda_{\alpha} / \sqrt{ }(1+\varepsilon) \approx \lambda_{\alpha} / 1.15 \approx$ $0.87 \lambda_{\alpha} \approx 1.09 \mathrm{~m}>1$ for all $\mathrm{m}$ and hence all the harmonics $\omega \approx m \omega_{c \alpha}$ will have approximately the same growth rate due to these boundary alphas, $Y_{m b \alpha}=\left[V_{\perp d e} J_{m}^{\prime}\left(\lambda_{d e}\right) / V_{\perp b \alpha} J_{m}\left(\lambda_{b \alpha}\right)\right]^{2}$ $\leq Y_{1 b \alpha}=\left(\lambda_{\mathrm{de}} / \lambda_{\mathrm{b} \alpha}\right)^{2} \approx 7.6 \times 10^{-4}$ and since $\lambda_{\mathrm{deJ}_{\mathrm{m}}}\left(\lambda_{\mathrm{de}}\right) \approx\left(\lambda_{\mathrm{de}} / 2\right)^{m} /(\mathrm{m}-1) !, Y_{m b \alpha} \approx$ $(0.03 / 2)^{2(m-1)} Y_{1 b \alpha}$. That is, $Y_{m b \alpha}$ decreases rapidly as $m$ increases since the damping rate of the higher harmonics by the background deuterium plasma ions decreases rapidly as $m$ increases for $\lambda_{\text {de }} \ll 1$. As we pointed out earlier, $v_{\alpha c p h}=V_{d c p h}$ can take almost any value. However, the dominant wave-particle interactions occur in the edge plasma when $v_{\alpha c p h} \approx$ $V_{d c p h} \approx\left(2 \kappa T_{d e} / M_{d}\right)^{1 / 2}$. Then the necessary condition for the edge radiative instability of Eq. (8) becomes that $V_{\alpha} \geq \sqrt{ }\left(1+\varepsilon^{-1}\right)\left(2 \kappa T_{d e} / M_{d}\right)^{1 / 2} \approx 2\left(2 \kappa T_{d e} / M_{d}\right)^{1 / 2}$ which is certainly true for the newly born fusion alphas in TFTR (see reference [4]). This may explain why the ICE harmonics at $\omega \approx m \omega_{c \alpha} \approx m \omega_{c d}$ are observed immediately after the beams are turned on. 
Since half the edge population is carbon [4], then the second condition of Eq. (9) for sufficiency to induce this radiative instability become $\eta_{\alpha} \geq(0.015)^{2(m-1)} \times 0.23 \times 10^{-3}$ $\left[\kappa T_{\| b \alpha}(t) / \kappa T_{d e}\right] /\left\{(1 / 2)\left[E_{\alpha} / k T d_{e}\right]^{1 / 2}-1\right\} \approx(0.015)^{2(m-1)} \times 0.46 \times 10^{-3}$ $\left[\kappa T_{\| b \alpha}(t) / \kappa T_{d e}\right]\left[\kappa T_{d e} / E_{\alpha}\right]^{1 / 2} \approx(0.015)^{2(m-1)} \times 2.8 \times 10^{-2}\left[\kappa T_{\| b \alpha}(t) / E_{\alpha}\right]$. Initially, at $t=0$, the fusion alphas are born monoenergetic and hence $T_{\| b \alpha}(t=0) \approx 0$ \{and indeed up to times $t$ such that $\left.\left[K T_{\| b \alpha}(t) / E_{\alpha}\right] \leq 0.11\right\}$ this condition is satisfied for all the harmonics $m$. But when $\left[k T_{\| b \alpha}(t) / E_{\alpha}\right]>0.11$, this condition cannot be satisfied for $m=1$, but is again satisfied for $m \geq 2$. From [4] we find that $\left[K T_{\| b \alpha}(t) / E_{\alpha}\right] \approx 0.11$ when $t \approx 90 \mathrm{~ms}$. We should point out that strictly speaking the observed alpha particle ICE seems to originate at 4 to $5 \mathrm{~cm}$ beyond the limitter in the scrape-off layer. In the scrape-off layer both the density and temperature are e-folding rapidly down to lower values, and hence the actual values of the edge $n_{d e}$ and $T_{d e}$ could be much lower than the values used here. This will raise the actual value of $\left[K T_{\| b \alpha}(t) / E_{\alpha}\right]$ at which the instability is quenched, and thus will raise the theoretically expected value of $\tau_{i c e}$ accordingly. The alpha particle slowing-down time from plasma center to edge [2] $\tau_{S \alpha} \approx 650$ to $130 \mathrm{~ms}$. Thus, theoretically we expect this radiative instability, and consequently, the associated alpha particle ICE to be quenched for times $t>\tau_{S \alpha} \approx 130$ to 650 $\mathrm{ms}$, since these trapped-passing boundary alpha particles making their fattest banana orbits spend part of their time near the plasma center and another part near the plasma edge.

In conclusion, we find that both the experimentally observed time behavior and the spatially localized origin of the fusion alpha particle ICE in TFTR are reasonably consistent with the theoretical predictions based on two-stream cyclotron radiative instabilities induced in the region near the outer midplane plasma edge by the marginally mirror trapped fraction of the newly born fusion alphas. Since theoretically the slowing-down distribution is radiatively stable, one expects the unstable ICE in the time interval $0 \leq t<\tau_{S \alpha}$, the fusion alphas 
slowing-down time, and this is roughly consistent with the observed $\tau_{i c e}$. Eventhough we have not done any nonlinear calculation of the saturated level of emission, since for these alphas $k_{\perp} \rho$ $>1$ for all harmonics, it is apparent that their linear growth rates will be roughly the same for all the harmonics. If the nonlinear saturated levels are proportional to their linear growth rates (which according to Coppi,et.al. $[15,6]$, is true theoretically if the dominant nonlinear saturation mechanism is the nonlinear mode coupling), then the experimentally observed equal amplitude harmonic ICE is consistent with the theoretical expectations.

This work was supported by the U.S. Department of Energy under Contract No. DE-ACO2-76CHO3073. The author thanks his experimental and theoretical colleagues (in particular, R.V. Budny, G. Rewoldt, R. Majeski, N. Bretz, and S. Cauffman) for useful comments. 


\section{REFERENCES}

[1] J. D. Strachan et al., Phys. Rev. Lett. 72 (1994) 3526; R.J. Hawryluk et.al., Phys. Rev. Lett. 72 (1994) 3530.

[2] R.V. Budny, Nucl. Fusion 34 (1994) 1247.

[3] S. Cauffman and R. Majeski, Rev. Sci. Instrum. 66, 817 (1995); S. Cauffman, R. Majeski, K.G. McClements, and R.O. Dendy, Submitted to Nucl. Fusion (private communication).

[4] According to the TRANSP code plasma analysis of reference 2, the TFTR supershots edge background plasmas consists of about $50 \%$ deuterium ions and $50 \%$ fully ionized carbon impurity ions. The charge to mass ratio of these fully ionized carbon ions is equal to that of the deuterium ions and thus their cyclotron frequencies are degenerate (i.e., equal to each other). Also in this plasma edge region, the temperatures of the carbon impurity ions and the deuterium ions are equal to each other. That is, $k_{\perp} \rho$ of carbon $\approx(1 / 12)^{1 / 2}$ $k_{\perp} \rho$ of deuterium. Thus the damping of the fast Alfven waves at $\omega \approx m \omega_{c \alpha} \approx m \omega_{c d} \approx m \omega_{c c}$ by carbon $\approx(1 / 12)^{m}$ of that by deuterium. Hence, for our purposes here, without any loss of generality, we can take the entire background edge plasma as consisting of deuterium ions only but with half the edge population, since the damping contribution by carbon can be neglected for $k=k_{\perp}$. Furthermore, this TRANSP code analysis shows that the early time evolution of the fusion alphas velocity distribution function can be approximated by a drifting Maxwellian distribution with a fractional width $\left(\Delta E_{\alpha} / E_{\alpha}\right)=$ $2\left(\Delta V_{\alpha} / V_{\alpha}\right)$ increasing almost linearly with time from the value zero to 0.5 in the first $0.4 \mathrm{~s}$, and eventually reaching the slowing-down distribution function for $t \approx \tau_{s} \alpha$. However, once this fractional width has increased beyond 0.3 , it is not much meaningful to approximate it by a drifting Maxwellian, since the distribution is already on its way well towards a slowing-down type. (Also R.V. Budny and G. Rewoldt, private communication). 
[5] J. A. Rome, D. G. Mc Alees, J. D. Callen, and R. H. Fowler, Nucl. Fusion 16 (1976) 55.

[6] B. Coppi, Phys. Lett. A 172 (1993) 439; B. Coppi et.al., Phys. Fluids 29 (1986) 4060.

[7] N.N. Gorelenkov and C.Z. Cheng, Phys. Plasmas 2 (1995) 1.

[8] R.O. Dendy, et. al., Phys. Plasmas 1, (1994) 1918; K.G McClements, R.O Dendy, C.N. Lashmore-Davis, G.A. Cottrell, S. Cauffman, and R. Majeski, Submitted to Nucl. Fusion (private communication)

[9] J. Wesson, Tokamaks (Clarendon, Oxford, 1987).

[10] V. Arunasalam, G. J. Greene, and K. M. Young, Nucl. Fusion 34 (1994) 927.

[11] V. Arunasalam, Phys. Fluids B 4 (1992) 1643; A. A. Rukhadze and V. P. Silin, Sov. Phys.-Tech. Phys. (Eng. Trans.) 7 (1962) 307.

[12] S. Glasstone and R. H. Lovberg, Controlled Thermonuclear Reactions (Van Nortrand, New York, 1960).

[13] T. E. Stringer, Plasma Phys. 16 (1974) 651.

[14] G. A. Cottrell et al., Nucl. Fusion 33 (1993) 1365.

[15] B. Coppi, M.N. Rosenbluth, and R.N. Sudan, Ann. Phys. 55 (1969) 193. 
Dr. F. Paoloni, Univ. of Wollongong, AUSTRALIA

Prof. R.C. Cross, Univ. of Sydney, AUSTRALIA

Plasma Research Lab., Australian Nat. Univ., AUSTRALIA

Prof. I.R. Jones, Flinders Univ, AUSTRALIA

Prof. F. Cap, Inst for Theoretical Physics, AUSTRIA

Prof. M. Heindler, Institut für Theoretische Physik, AUSTRIA

Prof. M. Goossens, Astronomisch Instituut, BELGIUM

Ecole Royale Militaire, Lab. de Phy. Plasmas, BELGIUM

Commission-European, DG. XII-Fusion Prog., BELGIUM

Prof. R. Bouciqué, Rijksuniversiteit Gent, BELGIUM

Dr. P.H. Sakanaka, Instituto Fisica, BRAZIL

Prof. Dr. I.C. Nascimento, Instituto Fisica, Sao Paulo, BRAZIL

Instituto Nacional De Pesquisas Espaciais-INPE, BRAZIL

Documents Office, Atomic Energy of Canada Ltd., CANADA

Ms. M. Morin, CCFMTokamak de Varennes, CANADA

Dr. M.P. Bachynski, MPB Technologies, Inc., CANADA

Dr. H.M. Skarsgard, Univ. of Saskatchewan, CANADA

Prof. J. Teichmann, Univ. of Montreal, CANADA

Prof. S.R. Sreenivasan, Univ. of Calgary, CANADA

Prof. R. Marchand, INRS-Energie et Materiaux, CANADA

Dr. R. Boiton, Centre canadien de fusion magnétique, CANADA

Dr. C.R. James, Univ. of Alberta, CANADA

Dr. P. Lukác, Komenského Universzita, CZECHO-SLOVAKIA

The Librarian, Culham Laboratory, ENGLAND

Library, R61, Rutherford Appleton Laboratory, ENGLAND

Mrs. S.A. Hutchinson, JET Library, ENGLAND

Dr. S.C. Sharma, Univ. of South Pacific, FIJI ISLANDS

P. Mähönen, Univ. of Helsinki, FINLAND

Prof. M.N. Bussac, Ecole Polytechnique, FRANCE

C. Mouttet, Lab. de Physique des Milieux lonisés, FRANCE

J. Radet, CEN/CADARACHE - Bat 506, FRANCE

Prof. E. Economou, Univ. of Crete, GREECE

Ms. C. Rinni, Univ. of loannina, GREECE

Preprint Library, Hungarian Academy of Sci., HUNGARY

Dr. B. DasGupta, Saha Inst. of Nuclear Physics, INDIA

Dr. P. Kaw, Inst. for Plasma Research, INDIA

Dr. P. Rosenau, Israel Inst of Technology, ISRAEL

Librarian, Intemational Center for Theo Physics, ITALY

Miss C. De Palo, Associazione EURATOM-ENEA, ITALY

Dr. G. Grosso, Istituto di Fisica del Plasma, ITALY

Prof. G. Rostangni, Istituto Gas Ionizzati Del Cnr, ITALY
Dr. H. Yamato, Toshiba Res \& Devel Center, JAPAN

Prof. I. Kawakami, Hiroshima Univ., JAPAN

Prof. K. Nishikawa, Hiroshima Univ., JAPAN

Librarian, Naka Fusion Research Establishment, JAERI, JAPAN

Director, Japan Atomic Energy Research Inst., JAPAN

Prof. S. Itoh, Kyushu Univ., JAPAN

Research Info. Ctr., National Instit. for Fusion Scienć, JAPAN

Prof. S. Tanaka, Kyoto Univ., JAPAN

Library, Kyoto Univ., JAPAN

Prof. N. Inove, Univ. of Tokyo, JAPAN

Secretary, Plasma Section, Electrotechnical Lab., JAPAN

Dr. O. Mitarai, Kumamoto Inst. of Technology, JAPAN

Dr. G.S. Lee, Korea Basic Sci. Ctr, KOREA

J. Hyeon-Sook, Korea Atomic Energy Research Inst., KOREA

D.I. Choi, The Korea Adv. Inst. of Sci. \& Tech., KOREA

Leandro Melendez Lugo, Inst. Nac1. de Inves. NuCl, MEXICO

Prof. B.S. Liley, Univ. of Waikato, NEW ZEALAND

Inst of Physics, Chinese Acad Sci PEOPLE'S REP. OF CHINA

Library, Inst. of Plasma Physics, PEOPLE'S REP. OF CHINA

Tsinghua Univ. Library, PEOPLE'S REPUBLIC OF CHINA

Z. Li, S.W. Inst Physics, PEOPLE'S REPUBLIC OF CHINA

Prof. J.A.C. Cabral, Instituto Superior Tecnico, PORTUGAL

Prof. M.A. Hellberg, Univ. of Natal, S. AFRICA

Prof. D.E. Kim, Pohang Inst. of Sci. \& Tech., SO. KOREA

Prof. C.I.E.M.A.T, Fusion Division Library, SPAIN

Dr. L. Stenflo, Univ. of UMEA, SWEDEN

Library, Royal Inst. of Technology, SWEDEN

Prof. H. Wilhelmson, Chalmers Univ. of Tech., SWEDEN

Centre Phys. Des Plasmas, Ecole Polytech, SWITZERLAND

Bibliotheek, Inst. Voor Plasma-Fysica, THE NETHERLANDS

Asst. Prof. Dr. S. Cakir, Middle East Tech. Univ., TURKEY

Dr. V.A. Glukhikh,Sci. Res. Inst. Electrophys.I Apparatus, USSR

Dr. D.D. Ryutov, Siberian Branch of Academy of Sci., USSR

Dr. G.A. Eliseev, I.V. Kurchatov Inst., USSR

Librarian, The Ukr.SSR Academy of Sciences, USSR

Dr. L.M. Kovrizhnykh, Inst. of General Physics, USSR

Kernforschungsanlage GmbH, Zentralbibliothek, W. GERMANY

Bibliothek, inst. Fü Plasmaforschung, W. GERMANY

Prof. K. Schindler, Ruhr-Universitát Bochum, W. GERMANY

Dr. F. Wagner, (ASDEX), Max-Planck-institut, W. GERMANY

Librarian, Max-Planck-Institut, W. GERMANY 